\title{
MATRIX REPRESENTATIONS OF ARTIN GROUPS
}

\author{
CRAIG C. SQUIER
}

(Communicated by Bhama Srinivasan)

\begin{abstract}
We define matrix representations of Artin groups over a 2-variable Laurent-polynomial ring and show that in the rank 2 case, the representations are faithful. In the special case of Artin's braid group, our representation is a version of the Burau representation and our faithfulness theorem is a generalization of the well-known fact that the Burau representation of $B_{3}$ is faithful.
\end{abstract}

In [4], Brieskorn and Saito coined the phrase "Artin groups" to denote a certain class of groups, defined by generators and relations, which stand in relationship to arbitrary Coxeter groups much as Artin's braid group $B_{n}[\mathbf{1}]$ stands in relationship to the symmetric group $S_{n}$. One of the nice features of Coxeter groups is that they have "standard" representations [6] as groups of matrices over the real numbers preserving a suitably defined bilinear form and that, moreover, these representations are faithful (see [3]). Our purpose here is to show the existence of analogous matrix representations of Artin groups over Laurent-polynomial rings preserving similarly defined sequilinear forms. Unfortunately, except in the simplest cases, the question of faithfulness of these Artin group representations remains open.

In $\S 1$, we define Artin groups $G_{M}$ (by representation), a Hermitian form $J$, and unitary reflections for each given generator of $G_{M}$; these are defined using a given Coxeter matrix $M$. In $\S 2$, we show that the reflections associated to generators of $G_{M}$ define a matrix representation of $G_{M}$ (Theorem 1) and that when the presentation of $G_{M}$ involves 2 generators, this representation is faithful (Theorem 2). We note that in the special case of the braid groups our representation is a version of the Burau representation ([5] or see [2]). The results below are first, a generalization to arbitrary Artin groups of the author's observation [10] that the Burau representation of $B_{n}$ is unitary and second, a generalization to arbitrary rank 2 Artin groups of the well-known fact (see [9 or 2]) that the Burau representation of $B_{3}$ is faithful.

1. Definitions. Let $n$ be a positive integer. A (rank $n$ ) Coxeter matrix $M$ will be an $n \times n$ symmetric matrix $M=[m(i, j)]$ each of whose entries $m(i, j)$ is a positive integer or $\infty$ such that $m(i, j)=1$ if and only if $i=j$. Out of a Coxeter matrix $M$, we shall build some presentations and some forms.

To define the presentations, let $X=\left\{x_{1}, \ldots, x_{n}\right\}$ be a finite set. For $m$ a positive integer, define the symbol $\langle x y\rangle^{m}$ by the formula

$$
\langle x y\rangle^{m}= \begin{cases}(x y)^{k} & \text { if } m=2 k, \\ (x y)^{k} x & \text { if } m=2 k+1 .\end{cases}
$$

Received by the editors February 5, 1987.

1980 Mathematics Subject Classification (1985 Revision). Primary 20F36; Secondary 20H10.

(C) 1988 American Mathematical Society $0002-9939 / 88 \$ 1.00+\$ .25$ per page 
Let $M$ be an $n \times n$ Coxeter matrix. $G_{M}$ will denote the abstract group defined by generators $X=\left\{x_{1}, \ldots, x_{n}\right\}$ and relations all $\left\langle x_{i} x_{j}\right\rangle^{m(i, j)}=\left\langle x_{j} x_{i}\right\rangle^{m(i, j)}$ for $1 \leq i<j \leq n$. Throughout, the case $m(i, j)=\infty$ will stand for "no relation". $G_{M}$ is the Artin group determined by $M . W_{M}$ will denote $G_{M}$ modulo the addition relations all $x_{i}^{2}=1$. Note that in the presence of the relations $x_{i}^{2}=1$, the defining relations of $G_{M}$ take the form $\left(x_{i} x_{j}\right)^{m(i, j)}=1$. $W_{M}$ is called the Coxeter group determined by $M$. For the basic properties of Coxeter groups, see [3 or 6]. For a study of Artin groups and their relationship to Coxeter groups, see [4].

We define a symmetric bilinear form $J_{1}$ associated to $W_{M}$ and a Hermitian form $J$ associated to $G_{M}$. To motivate the definitions of $J$, we begin by recalling the (well-known see [3]) definition of $J_{1}=J_{1}(M): J_{1}$ is the $n \times n$ matrix $\left[c_{i j}\right]$ where $c_{i j}=-2 \cos (\pi / m(i, j))$. Here, we adopt the convention that $\pi / \infty=0$ so that if $m(i, j)=\infty$ then $c_{i j}=-2$. Note that each $c_{i i}=2$. Let $V$ denote an $n$-dimensional vector space over $\mathbf{R}$ with basis $\left\{e_{1}, \ldots, e_{n}\right\}$. Identify each $v \in V$ with the column vector consisting of the coordinates of $v$ with respect to the basis $\left\{e_{1}, \ldots, e_{n}\right\}$ of $V$. With this convention, if $v \in V$, let $v^{\prime}$ denote the transpose of $v$ and, for $u, v \in V$, define $\langle u, v\rangle_{1}=u^{\prime} J_{1} v$. Thus, $J_{1}$ defines a symmetric bilinear form on $V$. We use $J_{1}$ to define a matrix representation $\rho_{1}$ of $W_{M}$ on $V$ : if $v \in V$ and $x_{i} \in X$ define

$$
\left(\rho_{1}\left(x_{i}\right)\right)(v)=v-\left\langle e_{i}, v\right\rangle_{1} e_{i} .
$$

It is well known (again see [3]) that $\rho_{1}$ is a faithful linear representation of $W_{M}$.

To define $J$, let $\Lambda$ denote the Laurent-polynomial ring $\mathbf{R}\left[s, s^{-1}, t, t^{-1}\right]$, where $s$ and $t$ are indeterminates over $\mathbf{R}$. Define $J=J(M)$ to be the $n \times n$ matrix $\left[a_{i j}\right]$ over $\Lambda$, where

$$
a_{i j}= \begin{cases}-2 s \cos (\pi / m(i, j)), & i<j, \\ 1+s t, & i=j, \\ -2 t \cos (\pi / m(i, j)), & i>j .\end{cases}
$$

Note that $J_{1}$ may be obtained from $J$ by substituting $s=t=1$.

To define analogues of the representation $\rho_{1}$ of $W_{M}$ defined above, we introduce an analogue of complex conjugation in the Laurent-polynomial ring $\Lambda$ : if $x \in \mathbf{R}$ then, as usual, $\bar{x}=x$; also, $\bar{s}=s^{-1}$ and $\bar{t}=t^{-1}$, extended to $\Lambda$ additively and multiplicatively. Note that if complex numbers of norm 1 are substituted for $s$ and $t$ then we recover ordinary complex conjugation.

We extend the definition of conjugation to matrices entrywise and, if $A$ is a matrix over $\Lambda$, we define $A^{*}=\bar{A}^{\prime}$. For example, note that $J^{*}=s^{-1} t^{-1} J$.

Let $V$ denote a free $\Lambda$-module with basis $\left\{e_{1}, \ldots, e_{n}\right\}$ and, as above, identify each $v \in V$ with its column vector of coordinates. If $u, v \in V$ define $\langle u, v\rangle=u^{*} J v$. Finally, we define $\rho$ : if $v \in V$ and $x_{i} \in X$ define

$$
\left(\rho\left(x_{i}\right)\right)(v)=v-\left\langle e_{i}, v\right\rangle e_{i} .
$$

We shall see below that $\rho$ provides a matrix representation of the Artin group $G_{M}$.

Note that $\left(\rho\left(x_{i}\right)\right)\left(v-s^{-1} t^{-1}\left\langle e_{i}, v\right\rangle e_{i}\right)=v$. It follows that each $\rho\left(x_{i}\right)$ acts invertibly on $V$. In fact, each $\rho\left(x_{i}\right)$ is a pseudo-reflection in the sense of [3]. Also, for each $x_{i} \in X$ and each $u, v \in V$, we have

$$
\left\langle\rho\left(x_{i}\right)(u), \rho\left(x_{i}\right)(v)\right\rangle=\langle u, v\rangle .
$$

Combining this observation with Theorem 1 below, we conclude that $\rho$ is a representation of $G_{M}$ in a group of unitary matrices. 
2. Theorems. In this section, we show that the function $\rho$ defined (on generators) above extends to a representation of the Artin groups $G_{M}$ and that when $n=2$, this representation is faithful. (The second result includes the fact that the Burau representation of $B_{3}$ is faithful-see [ 9 or 2].)

To prove that $\rho$ defines a representation of $G_{M}$, we need to show that $\rho$ respects the defining relations of $G_{M}$. An important observation is the following

LEMMA. $\operatorname{det} J \neq 0$.

PROOF. In $\operatorname{det} J$, the coefficients of $(s t)^{n}$ is 1 , so $\operatorname{det} J \neq 0$.

In particular, $\langle-,-\rangle$ is nondegenerate: if $u \in V$ satisfies $\langle u, v\rangle=0$ for all $v \in V$, then $u=0$.

At this point, it is convenient to introduce the field-of-quotients $F$ of $\Lambda . F$ is a rational function field over $\mathbf{R}$. Extend the definition of conjugation to $F$. Letting $V_{F}$ denote the $F$-vector space $V \otimes_{\Lambda} F$, extend $\langle-,-\rangle$ to $V_{F}$ and also view $\rho$ as a linear transformation on $V_{F}$. Note that since $\langle-,-\rangle$ is nondegenerate, if $u \in V_{F}$ satisfies $u \neq 0$, then $u^{\perp}=\left\{v \in V_{F} \mid\langle u, v\rangle=0\right\}$ is an $(n-1)$-dimensional subspace of $V_{F}$. Also note that $\rho\left(e_{i}\right)$ is the identity on $e_{i}^{\perp}$. Given $i, j$ satisfying $1 \leq i<j \leq n$, let $V_{i j}$ denote the subspace of $V_{F}$ spanned by $e_{i}$ and $e_{j}$, and let $V_{i j}^{\perp}=e_{i}^{\perp} \cap e_{j}^{\perp}$. We need the following

LEMMA. $V_{i j} \cap V_{i j}^{\perp}=\{0\}$.

PROOF. Let $v=v_{i} e_{i}+v_{j} e_{j} \in V_{i j}$ where $v_{i}, v_{j} \in \Lambda$. If $v \in V_{i j}^{\perp}$, then $\left\langle e_{i}, v\right\rangle=$ $\left\langle e_{j}, v\right\rangle=0$ which leads to the following system of linear equations:

$$
\begin{aligned}
v_{i}(1+s t)-2 v_{j} s \cos (\pi / m) & =0, \\
-2 v_{i} t \cos (\pi / m)+v_{j}(1+s t) & =0,
\end{aligned}
$$

where $m$ denotes $m(i, j)$. Since the determinant of the coefficient matrix is $\neq 0$ in $\Lambda$, the only solution is $v_{i}=v_{j}=0$, so $v=0$, as required.

Noting that the defining relations of $G_{M}$ each involve exactly two generators, in order to show that $\rho$ respects the defining relations of $G_{M}$, it suffices to show that each $\left\langle x_{i} x_{j}\right\rangle^{m(i, j)}=\left\langle x_{j} x_{i}\right\rangle^{m(i, j)}$ holds under $\rho$ on the subspace $V_{i j}$ of $V_{F}$.

Let $a$ denote the matrix of $x_{i}$ and $b$ the matrix of $x_{j}$ with respect to the basis $e_{i}, e_{j}$ of $V_{i j}$. Writing $m$ for $m(i, j)$, it follows that

$$
a=\left(\begin{array}{cc}
-s t & 2 s \cos (\pi / m) \\
0 & 1
\end{array}\right), \quad b=\left(\begin{array}{cc}
1 & 0 \\
2 t \cos (\pi / m) & -s t
\end{array}\right) \text {. }
$$

Thus it suffices to prove

LEMMA. The matrices $a$ and $b$ above satisfy $\langle a b\rangle^{m}=\langle b a\rangle^{m}$.

PROOF. Adjoin a square root $q$ of $s t^{-1}$ to $F$ and let

$$
R=\left(\begin{array}{cc}
0 & q \\
q^{-1} & 0
\end{array}\right)
$$

It is easy to check that $R^{2}=I$ and $b=R a R$. It follows that $\langle a b\rangle^{m}=\langle b a\rangle^{m}$ if and only if $(a R)^{m}=(R a)^{m}$. Clearly, $s^{-1} q(a R)$ and $s^{-1} q(R a)$ have determinant 1 and trace $2 \cos (\pi / m)$. It follows that $\left(s^{-1} q(a R)\right)^{m}=\left(s^{-1} q(R a)\right)^{m}=-I$, as required.

Thus we have the following theorem. 
THEOREM 1. The function $\rho$ extends to a representation of $G_{M}$ in $G L_{n}(\Lambda)$.

PROOF. Each relation $\left\langle x_{i} x_{j}\right\rangle^{m(i, j)}=\left\langle x_{j} x_{i}\right\rangle^{m(i, j)}$ holds under $\rho$ on $V_{i j}$ by the lemma and therefore on all of $V_{F}$ since $x_{i}$ and $x_{j}$ are each the identity on $V_{i j}^{\perp}$.

Except in the two-generator case, we do not know if the representation $\rho$ is faithful. Here is the proof in the two-generator case. Let $A$ and $B$, respectively, denote the matrices obtained by substituting $s=1$ and $t=-1$ in $a$ and $b$ above.

LEMMA. The matrix group generated by $A$ and $B$ has presentation $\langle A B\rangle^{m}=$ $\langle B A\rangle^{m}$ and

$$
\begin{aligned}
(A B)^{m} & =1 & & (m \text { even }), \\
(A B)^{2 m} & =1 & & (m \text { odd }) .
\end{aligned}
$$

ProOF. View $A$ and $B$ as linear fractional transformations acting on the upper half-plane. Using the fact that the matrix $A B$ has determinant 1 and trace $2 \cos (\pi(1-(2 / m)))$, it follows that $A B$ satisfies $(A B)^{m}=(-1)^{m} I$. Thus, it suffices to prove that the group of linear fractional transformations generated by $A$ and $B$ has defining relations $\langle A B\rangle^{m}=\langle B A\rangle^{m}$ and $(A B)^{m}=1$.

We prove this last fact by exhibiting the group generated by $A$ and $B$ as a subgroup of finite index in a suitable triangle group. Let $R_{1}, R_{2}$ and $R_{3}$ be transformations of the upper half-plane defined by

$$
\begin{aligned}
& R_{1}=\text { reflection in the imaginary axis } x=0, \\
& R_{2}=\text { reflection in the axis } x=\cos (\pi / m), \\
& R_{3}=\text { reflection in the unit circle. }
\end{aligned}
$$

Then $R_{1}, R_{2}$ and $R_{3}$ generate a $(2, m, \infty)$ triangle group with presentation (see [7]):

$$
R_{1}^{2}=R_{2}^{2}=R_{3}^{2}=\left(R_{1} R_{3}\right)^{2}=\left(R_{2} R_{3}\right)^{m}=1 .
$$

Noting that $R_{1}(z)=-\bar{z}, R_{2}(z)=-\bar{z}+2 \cos (\pi / m)$ and $R_{3}(z)=1 / \bar{z}$, it follows that, as linear fractional transformations, $A=R_{2} R_{1}$ and $B=R_{3} R_{1} R_{2} R_{3}$. It can be checked that the subgroup of the triangle group generated by $A$ and $B$ is normal and has index 2 when $m$ is odd and index 4 when $m$ is even. A routine application of the Reidemeister-Schreier algorithm produces the required presentation of the group generated by $A$ and $B$.

THEOREM 2. The group of matrices generated by $a$ and $b$ has presentation $\langle a b\rangle^{m}=\langle b a\rangle^{m}$.

PROOF. By the Lemma, the substitution produces a group with a presentation consisting of the desired relation together with a further relation $c=1$ where $c=(a b)^{m}$ when $m$ is even and $c=(a b)^{2 m}$ when $m$ is odd. In either case, $c$ is a central element in the group defined by $\langle a b\rangle^{m}=\langle b a\rangle^{m}$. It follows that any additional relation between $a$ and $b$ must be a nonzero power of $c$. But any nonzero power of $c$ has determinant a nonzero power of $-s t$ and is therefore not the identity. Thus the matrix group generated by $a$ and $b$ has presentation $\langle a b\rangle^{m}=\langle b a\rangle^{m}$, as desired. 
3. Remarks. The (reduced) Burau representation of $B_{n}$ (see [2]) may be obtained by substituting $s=1$ in the representation $\rho$ of $B_{n}$ that arises above. In fact, the representation $\rho$ itself is equivalent to the Burau representation: it is possible to conjugate the image of $\rho$ by a diagonal matrix that, in each $\rho\left(x_{i}\right)$, "moves the $t$ 's above the diagonal" and "leaves the $s$ 's alone". The matrices that result have the property that their entries depend only on the product st. A similar conjugation is possible whenever the Coxeter graph $\Gamma_{M}$ of $M$ is a forest $\left(\Gamma_{M}\right.$ has vertices $X$ and an edge connecting $x_{i}$ and $x_{j}$ provided $m(i, j) \geq 3$ ). In these cases, the representations $\rho$ of $G_{M}$ is conjugate to a representation over the Laurent-polynomial ring $\mathbf{R}\left[s t,(s t)^{-1}\right] \subseteq \Lambda$. In the case of $B_{n}$, the representation that results is the Burau representation.

In general, the question of the faithfulness of $\rho$ remains open. The only known cases seem to be those that follow easily from Theorem 2: $G_{M}$ is a direct product of rank 1 or 2 Artin groups (equivalently, $\Gamma_{M}$ is a disjoint union of vertices and pairs of vertices connected by an edge). Much effort has been devoted (unsuccessfully) to trying to determine whether or not the Burau representation of $B_{4}$ is faithful. One other case that might be worth investigating is $M$ defined by each $m(i, j)=\infty$, so that $G_{M}$ is a free group.

\section{REFERENCES}

1. E. Artin, Theorie der Zopfe, Abh. Math. Sem. Univ. Hamburg 9 (1925), 47-72.

2. J. Birman, Braids, links and mapping class groups, Ann. of Math. Studies, no. 82, Princeton Univ. Press, Princeton, N.J., 1974.

3. N. Bourbaki, Groupes et algebres de Lie, Chaps. 4-6, Elements de Mathematique 34, Paris, Hermann, 1968.

4. E. Brieskorn and K. Saito, Artin-Gruppen und Coxeter-Gruppen, Invent. Math. 17 (1972), 245-271. (German)

5. W. Burau, Über Zopfgruppen und gleichsinnig verdrillte Verkettunger, Abh. Math. Sem. Univ. Hanischen 11 (1936), 171-178.

6. H. S. M. Coxeter, Discrete groups generated by reflections, Ann. of Math. (2) 35 (1934), 588621 .

7. H. S. M. Coxeter and W. O. J. Moser, Generators and relations for discrete groups, SpringerVerlag, Berlin, 1957.

8. H. Hiller, Geometry of Coxeter groups, Pitman, Boston, Mass., 1982.

9. W. Magnus and A. Peluso, On a theorem of V. I. Arnol'd, Comm. Pure Appl. Math. 22 (1969), 683-692.

10. C. Squier, The Burau representation is unitary, Proc. Amer. Math. Soc. 90 (1984), 199-202.

Department of Mathematical Sciences, State University of New York at BinghaMTON, BinghaMTON, NEW YORK 13901 